Classcraft como herramienta gamificada para la enseñanza de Integración de procesos con tecnología informática

Classcraft as a gamified tool for the teaching of Integration of processes with computer technology

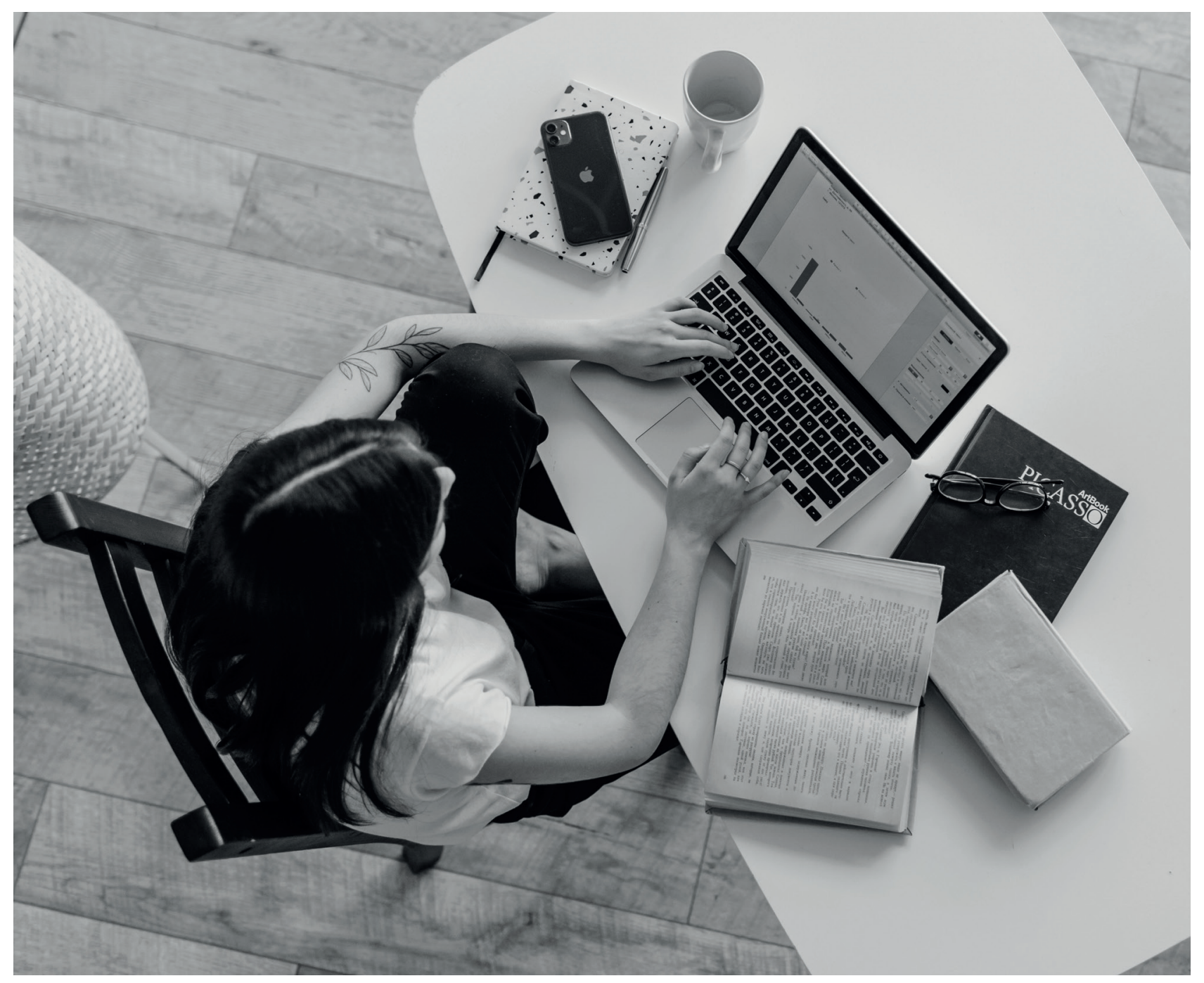

Fuente: Pexels.com Licencia Creative Commons 


\title{
Classcraft como herramienta gamificada para la enseñanza de Integración de procesos con tecnología informática ${ }^{1}$
}

\section{Classcraft as a gamified tool for the teaching of Integration of processes with computer technology}

\author{
Nicolás Hernández-Durán², Martha Liliana Torres-Barreto ${ }^{3}$, Mariana Acuña-Rangel ${ }^{4}$
}

Artículo recibido en marzo 24 de 2020; artículo aceptado en julio 23 de 2020

\begin{abstract}
Este artículo puede compartirse bajo la Licencia Creative Commons Atribución-NoComercial-Compartirlgual 4.0 Internacional y se referencia usando el siguiente formato: Hernández-Durán, N., Torres-Barreto, M. L y Acuña-Rangel, M. (2021). Classcraft como herramienta gamificada para la enseñanza de Integración de procesos con tecnología informática. I+D Revista de Investigaciones, 16(1), 62-74. http://dx.doi.org/10.33304/revinv.v16n1-2021006.
\end{abstract}

\begin{abstract}
Resumen
Las nuevas tecnologías continúan cambiando esquemas y formas de gestionar información de procesos industriales. Con ellas han surgido también cambios en la forma como la sociedad actual se relaciona, se comunica, cómo entiende las interacciones entre personas, cómo encuentra nuevas motivaciones asociadas al uso de la tecnología. Este trabajo se centra en estudiar la motivación generada en una clase de pregrado universitario a la cual se incorporó una herramienta gamificada estándar en el mercado para la enseñanza y el aprendizaje del curso. Los contenidos de la clase tienen relación directa con la integración de tecnología en los procesos de negocio, y los estudiantes incluidos en la muestra están en quinto año de un programa de ingeniería. Los resultados muestran que los estudiantes prefieren ampliamente una clase gamificada, pero que, al mismo tiempo, algunos de los elementos de la gamificación, entre ellos los que tienen que ver con efectos aleatorios, no son bien percibidos, mientras que los avatares dan sentido al desarrollo de una comunidad virtual con nuevas identidades personales, lo cual llama profundamente la atención de los jóvenes de educación media.
\end{abstract}

Palabras clave: Classcraft, educación, gamificación, procesos, tecnología.

\footnotetext{
1 Reporte de caso, investigación cualitativa, resultado de un proyecto de investigación culminado, perteneciente al área de ciencias sociales y sistemas de información, subárea de educación y dirección de operaciones, desarrollado en el Grupo de Investigación Finance \& Management, fue financiado por la Universidad Industrial de Santander (Bucaramanga, Colombia). Dirección: carrera 27 calle 9, campus universitario, PBX: 6344000. Fecha de inicio: enero de 2020. Fecha de terminación: abril del 2020.

${ }^{2}$ Estudiante de Ingeniería Industrial, Universidad Industrial de Santander. Auxiliar del Laboratorio Galea adscrito al Grupo de Investigación Finance \& Management, Universidad Industrial de Santander (Bucaramanga, Colombia). Dirección: carrera 27 calle 9, campus universitario, PBX: 6344000. ORCID ID: https://orcid.org/0000-0001-9754-9187. Correo electrónico institucional: nicolas.hernandez3@correo.uis.edu.co.

${ }^{3}$ Ph. D. en Estrategia y Marketing de la Empresa, Universidad De Castilla - La Mancha. Directora del Grupo de investigación Finance \& Management y del Laboratorio GALEA de la Universidad Industrial de Santander (Bucaramanga, Colombia). Dirección: carrera 27 calle 9, campus universitario, PBX: 6344000. ORCID ID: https://orcid.org/0000-0002-4388-5991. Correo electrónico institucional: mltorres@uis.edu.co.

4 Estudiante de Ingeniería Industrial, Universidad Industrial de Santander. Auxiliar del Laboratorio Galea adscrito al Grupo de Investigación Finance \& Management, Universidad Industrial de Santander (Bucaramanga, Colombia). Dirección: carrera 27 calle 9, campus universitario, PBX: 6344000. ORCID ID: https://orcid.org/0000-0001-9895-6809. Correo electrónico institucional: mariana.acuna@correo.uis.edu.co.
} 
Nicolás Hernández-Durán, Martha Liliana Torres-Barreto, Mariana Acuña-Rangel

Classcraft como herramienta gamificada para la enseñanza de Integración de procesos con tecnología informática

\begin{abstract}
New technologies continue to change schemes and ways of managing information from industrial processes. With them, changes in the way society today relates, communicates, how it understands the interactions between people, how it finds new motivations associated with the use of technology have also emerged. This work focuses on studying the motivation generated in a university undergraduate class, in which a standard gamified tool in the market was incorporated for the teaching and learning of the course. The contents of the class are directly related to the integration of technology into business processes, and the students included in the sample are in the fifth year of an engineering program. The results show that students widely prefer a gamified class, but, at the same time, some of the elements of gamification, including those related to random effects, are not well perceived, while the avatars give meaning to the development of a virtual community with new personal identities, which deeply appeals to young people in secondary education.
\end{abstract}

Keywords: Classcraft, education, gamification, processes, technology.

\section{Introducción}

La cantidad de información que se genera en un proceso de negocio es innegable. Si se piensa en toda la cadena de suministro, por ejemplo, el aprovisionamiento, la producción, la logística de distribución y el almacenamiento son procesos que continuamente están generando datos que necesitan ser almacenados, tratados y analizados para poder obtener de ellos información útil para quien toma decisiones. No obstante, considerando la cantidad ingente de información, los sistemas de apoyo, como los sistemas de información empresarial, tienen un papel muy relevante al permitir que todos los datos se capturen, que se procesen en información y que esta información esté disponible en el momento en que se necesite. Desde la ingeniería industrial, la gestión de la información asociada a estos procesos de negocios se trata en diversas asignaturas que tienen relación con la integración de procesos con tecnologías de la información. Estas asignaturas están diseñadas para inducir al estudiante a lo que puede ser un entorno productivo, mediado por tecnología informática.

Por su parte, desde la academia, los educadores tienen un llamado cada vez más acucioso a entender cómo aprende el cerebro, cómo los estudiantes procesan la información, cómo controlan sus emociones, sus sentimientos, sus estados conductuales; todo esto, requisito indispensable para la innovación pedagógica y la transformación de los sistemas educativos. De hecho, a medida que el conocimiento relacionado al funcionamiento del cerebro humano vaya siendo más accesible a los educadores, el proceso de aprendizaje se volverá más efectivo y significativo, tanto para el educador como para el alumno (Campos, 2010).

Con este contexto, diferentes esfuerzos están siendo realizados en materia de innovación educativa. Considerando que las motivaciones hacia el aprendizaje de la presente generación no son necesariamente los mismos que hace unos años, diversos ejercicios gamificados surgen en un contexto en el que la atención del estudiante y su interés por aprender están en constante evolución.

Este artículo responde a la pregunta de investigación: ¿En qué medida una herramienta gamificada puede apoyar el proceso de enseñanza y de aprendizaje de estudiantes de educación superior en lo que respecta a integración de procesos con tecnologías de información?

\section{Integración de estrategias en el aula}

En la educación tradicional, el docente puede emplear estrategias para la enseñanza y el aprendizaje que incluyen, por ejemplo, (1) estrategias de atención, orientadas a centrar al estudiante en la tarea de forma específica; (2) estrategias de codificación, elaboración y organización, que incluyen técnicas como el subrayado, resumen, esquema, mapas conceptuales, cuadros sinópticos, entre otros; (3) estrategias de repetición y almacenamiento, que controlan los procesos de retención y memoria a corto y largo plazo, y finalmente (4), estrategias de comunicación y uso de la información adquirida, que permiten la elaboración de informes, la realización de síntesis, entre otras (Morales y Alfonso, 2006).

A pesar de lo anterior, dichas estrategias se han quedado algo rezagadas, en la medida en que han ido surgiendo descubrimientos acerca del funcionamiento del cerebro. De hecho, parece ser que las funciones cognitivas del cerebro se activan en realidad a partir de interacciones entre las funciones del cerebelo, el lóbulo frontal, la corteza motora y los sistemas de atención (Ito, 2000), todas conjuntamente. De esta forma, las estrategias de enseñanza y de aprendizaje están llamadas a migrar hacia otras que incluyan más estímulos sensoriales que mantengan la atención de los estudiantes durante mayor tiempo a través de las interacciones cerebrales. Estímulos musicales, visuales, de posicionamiento geoespacial, entre otros, que avivan la percepción sensorial (Dunbar, 2008; Hardiman, 2003). 
Nicolás Hernández-Durán, Martha Liliana Torres-Barreto, Mariana Acuña-Rangel Classcraft como herramienta gamificada para la enseñanza de Integración de procesos con tecnología informática

Es en este punto donde la gamificación encuentra su espacio. Un entorno gráfico -como los gamificadospoblado de historias y recorridos, con escenarios de colaboración, competencia o recolección, donde los estímulos son abundantes y variados, tiene todo el potencial para ayudar a los estudiantes a hacer conexiones entre lo que aprendieron anteriormente y lo que acaban de aprender. Además, fomentan la memoria a largo plazo, ya que muchas de las experiencias de juego activan el sistema sensorial cinestésico, lo que hace que ese recuerdo quede claro en la memoria (Sousa, 2011).

Parte del éxito de las experiencias gamificadas radica precisamente en que la dinámica del juego estimula el hemisferio derecho del cerebro y ayuda a los estudiantes a percibir los conceptos en su totalidad y no solamente dentro de los patrones lingüísticos tradicionales (propios del hemisferio izquierdo), que eran $\tan$ comunes y prácticamente la única alternativa hasta hace algunos años (Frost y Eden, 2014; Glover, 2013).

\section{La teoría detrás de la gamificación}

La gamificación, también conocida en el idioma español como ludificación o en inglés como gamification, cuando se realiza la búsqueda de la evolución del concepto, es una herramienta de enseñanza y de aprendizaje que permite incorporar elementos de diversión y entretenimiento en contextos que no están relacionados con el juego, lo que permite mejorar la experiencia de los usuarios (Klock et al., 2019). No se trata solo de aplicar la tecnología a los viejos modelos de motivación, como otorgar medallas; la gamificación crea modelos de compromiso completamente nuevos, dirigidos a nuevas comunidades de personas, que las motiva a alcanzar objetivos que tal vez ni siquiera sepan que tienen (Acosta-Medina et al., 2020; Burke, 2016).

En la gamificación se usa una variedad de elementos de juego para obtener una respuesta llena de significado para los usuarios (Zichermann y Cunningham, 2011). En contextos educativos, la gamificación se convierte en una aplicación cuidadosa de todos los elementos de juego adecuados para fomentar el aprendizaje. Estos elementos se dividen en tres categorías, siguiendo su nivel de abstracción: componentes, mecánica y dinámica (Kapp, 2012).

Los componentes son instancias específicas de la mecánica y la dinámica, que se aplican efectivamente en el sistema (Werbach y Hunter, 2012). Algunos de ellos son el avatar, las insignias, los regalos, las tablas de clasificación, los niveles, las misiones, los puntos y los activos virtuales. En el siguiente nivel de abstracción está la mecánica, que es el proceso que estimula la acción y el compromiso del usuario, que se logra a través de los componentes (Werbach y Hunter, 2012), como, por ejemplo, los retos, las competencias, la personalización, la retroalimentación y las recompensas. La dinámica se encuentra en el nivel más alto de abstracción, y hace referencia a aspectos controlados por la gamificación, pero logrados a través de los otros elementos. Dentro de las dinámicas existentes se encuentra la emoción, el progreso, la relación o interacción, y las reglas.

La combinación de estos elementos aumenta la experiencia del usuario cuando interactúa con el juego, a la vez que promueve la motivación, la diversión y el compromiso (Marache-Francisco y Brangier, 2014):

Un cerebro en constante aprendizaje y desarrollo permite aprovechar todo el potencial de la gamificación. El cerebro para aprender necesita percibir y codificar una información (input) y para ello utiliza sus recursos multisensoriales, el cuerpo, la motivación y todos los conocimientos previos. (Campos, 2010, p. 9)

La gamificación ha encontrado así en el storytelling una forma poderosa para establecer una fuerte conexión con los estudiantes a través de una narrativa que atrae y conecta sus sentidos (Acosta-Medina et al., 2020), "en tanto que el educador vincule su práctica pedagógica al maravilloso mundo del sistema natural de aprendizaje del cerebro, contribuyendo significativamente con el promover, desarrollar y fortalecer la red de conexiones neuronales" (Estupiñan Ricardo et al., 2016).

\section{La integración de procesos con tecnología informática en el currículo: los sistemas ERP}

El desarrollo profesional de un ingeniero industrial implica que esteadquiera los conocimientos fundamentales delos procesos que se suceden en las empresas (Torres-Barreto, 2018), la forma como se integran entre sí en la actividad operacional y la interacción y habilidad de quien dirija o coordine las diversas actividades que conducen al logro de objetivos financieros, de producción, comerciales, de mercadeo y recursos humanos (Davenport y Short, 1990; Lobo-Rueda et al., 2020).

Es en este ejercicio que el ingeniero industrial es protagonista en la formulación de estrategias a desarrollarse por medio de la administración de sistemas ERP (Nikolopoulos et al., 2003). Estos sistemas son soportados por sistemas de información. Por tal motivo, es fundamental que el futuro ingeniero incursione en el aprendizaje de los sistemas ERP y adquiera herramientas básicas que fortalecerán su capacidad de liderazgo y competencia para enfrentarse al reto de la gestión y administración de operaciones y la aplicación de herramientas integradoras de procesos que demanda la operatividad de las compañías actualmente (Berchet y Habchi, 2005).

En diferentes universidades, estos conocimientos se integran al currículo mediante una asignatura electiva a partir del tercer o cuarto año de carrera. En la universidad 
Nicolás Hernández-Durán, Martha Liliana Torres-Barreto, Mariana Acuña-Rangel

Classcraft como herramienta gamificada para la enseñanza de Integración de procesos con tecnología informática

donde se realizó este estudio, esta asignatura se ofrece como electiva. El curso de Integración de procesos con tecnología informática está estructurado en ocho bloques temáticos, y cuenta con material docente previamente elaborado, tal como se presenta en la Tabla 1. Este material está compuesto por presentaciones de PowerPoint que ejemplifican la teoría, ejercicios en los cuales los estudiantes pueden poner en práctica algunos componentes relacionados con la teoría, casos de estudio que simulan una situación de la vida real en la cual los estudiantes deben hacer uso de cada uno de los componentes aprendidos en el bloque temático, y finalmente se proporciona material complementario, que consiste en recursos audiovisuales, algunos casos de estudio complementarios, como material de lectura y retos proporcionados por la plataforma ERP con la que cuenta la universidad. Cada bloque temático se desarrolla en un espacio de dos semanas. La asignatura tiene una intensidad horaria de cuatro horas presenciales y cinco de trabajo independiente por semana.

Tabla 1

Bloques temáticos de la asignatura Integración de procesos con tecnología informática en la universidad seleccionada

\begin{tabular}{|c|c|c|c|c|}
\hline Bloques temáticos & 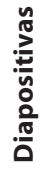 & 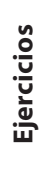 & 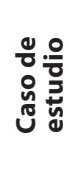 & 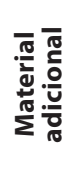 \\
\hline Sales and Distribution & 1 & 5 & 1 & 3 \\
\hline Materials Management & 1 & 5 & 1 & 1 \\
\hline Production Planning & 1 & 6 & 1 & 1 \\
\hline Finance & 1 & 4 & 1 & 1 \\
\hline Controling & 1 & 3 & 2 & 2 \\
\hline WareHouse Management & 1 & 2 & 4 & 4 \\
\hline Enterprise Asset Management & 1 & - & 1 & 1 \\
\hline Human Capital Management & 1 & - & 2 & 3 \\
\hline
\end{tabular}

Fuente: Autores.

En el módulo de Sales and Distribution se explican las bases que facilitan la gestión eficiente de todo lo que está relacionado con la venta y distribución de productos y servicios de una empresa o compañía a sus clientes. A través de este módulo, es posible la planificación e implementación efectiva de todas las acciones y tareas diarias vinculadas al ciclo de ventas de la empresa, como procesar los pedidos efectuados por los clientes, realizar precotizaciones, cotizaciones y gestionar los traslados y la entrega de los productos, suministrando y almacenando la información necesaria para relacionarse con los demás módulos del sistema ERP.

En el módulo de Materials Management se explican las bases que permiten a los estudiantes incursionar en la gestión de todo lo relativo a la contratación de servicios y la adquisición de bienes que se realizan en una organización. El módulo de gestión de materiales hace parte del sistema SAP, y ofrece solución a cada una de las diferentes etapas que componen la gestión de los materiales de una organización; es decir, desde la planificación y control de las necesidades, hasta la contratación de servicios, pasando por otras como la gestión de las compras, la recepción de mercancías y la gestión de inventarios.

Por su parte, en el módulo de Production Planning se explican todas las actividades que tienen lugar en la gestión de las instalaciones de producción de una organización. El objetivo fundamental de este módulo consiste en brindar a los estudiantes las herramientas suficientes para desarrollar competencias en las diferentes actividades que permitan asegurar que toda la producción sea realizada de forma efectiva, ajustando los procesos de producción a los tiempos de entrega que previamente han sido acordados con el cliente.

El módulo de Finance tiene como objetivo fundamental la gestión de todos los procesos contables y financieros de una organización. Gracias a este módulo, es posible realizar una revisión de la información financiera en tiempo real, algo de gran valor, ya que facilita no solo la toma de decisiones, sino que estas sean mucho más acertadas.

En el módulo de Controlling se explican las bases de cómo controlar, coordinar y optimizar todos y cada uno de los diferentes procesos de cálculo de costos en la organización. Este módulo es el encargado de la planificación contable de la compañía o empresa y, junto al módulo Finanzas, compone el sector de los módulos financieros del ERP.

En el módulo de Warehouse Management se explican las bases necesarias para comprender el funcionamiento de la gestión de los inventarios. Este módulo permite subdividir los almacenes de Gestión de Inventario en diferentes ubicaciones específicas $y$, al mismo tiempo, ofrece toda la funcionalidad relacionada con la entrada y salida de mercancías de estos, lo que permite gestionar operaciones de almacén de alto volumen e integrar la compleja logística de la cadena de suministro con los procesos de almacén y distribución.

En el módulo de Enterprise Asset Management se explican las bases para desarrollar competencias en la gestión de activos del negocio, lo que garantiza la disponibilidad y confiabilidad.

En toda buena gestión de un Activo hay tres fases: la fase de Proyecto, donde se planifica y se construye la instalación; la fase de Producción, donde se incluyen todas las tareas a realizar para obtener el producto, como Producción, Mantenimiento, Logística, Calidad y Gestión, y la última fase, que es la de Cierre de planta, donde se produce el desmantelamiento de esta. 
Finalmente, el módulo de Human Capital Management del ERP permite una gestión eficiente de la información y procesos del personal de una organización, e integra toda esta información y procesos con los demás módulos y con posibles aplicaciones externas.

\section{Metodología}

Para llevar a cabo el ejercicio lúdico se tomó como base la asignatura de Integración de procesos con tecnología informática, y la herramienta gamificada tipo open source Classcraft. Los pasos metodológicos seguidos se describen a continuación.

\section{Participantes y recursos}

Esta experiencia gamificada se llevó a cabo con estudiantes de ingeniería que cursaban la asignatura Integración de procesos con tecnología informática. Se tomaron dos grupos de noveno semestre (quinto año) de Ingeniería Industrial. En total fueron 43 participantes, divididos en grupos de 22 y 21 estudiantes cada uno. Adicionalmente participaron en la experiencia la profesora a cargo de la asignatura y dos auxiliares, uno de docencia y otro de investigación.

En materia de recursos, este ejercicio se llevó a cabo en aulas de cómputo de la universidad, donde se disponía de un ordenador para cada estudiante, así como de conexión a internet.

Para la validación de la experiencia se usó un cuestionario de 4 preguntas. Algunas de ellas diseñadas para contestar en un formato de escala Likert con valores de 1 a 5 y otras dicotómicas. El cuestionario se realizó teniendo en cuenta algunos lineamientos propuestos por Aji y Napitupulu (2018), y Rojas-López et al. (2019).

\section{Diseño de la experiencia}

Al diseñar la experiencia gamificada, el equipo del proyecto tuvo en cuenta una división de nueve pasos, de acuerdo con Sanabria (2006), tal como se muestra en la Figura 1. No obstante, para las condiciones particulares de este ejercicio, se realizaron adaptaciones que fueron válidas para el entorno colombiano y para la situación particular de los cursos seleccionados.

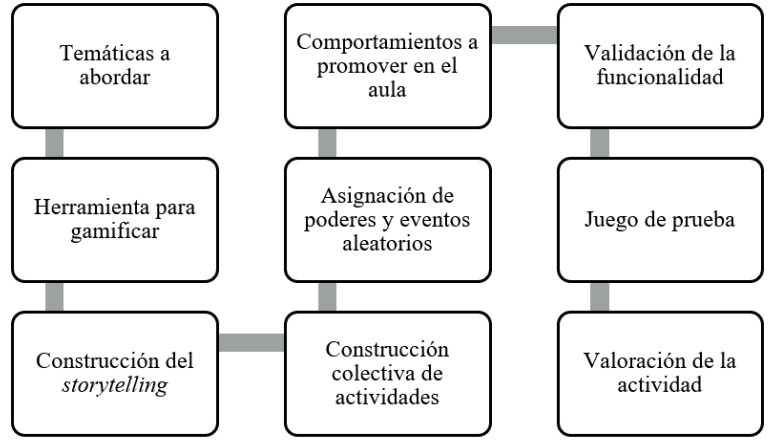

Figura 1. Pasos para el diseño de la experiencia gamificada. Fuente: Adaptación de Sanabria (2006).

\section{Identificación de las temáticas a abordar}

Gamificar el curso completo no era definitivamente una opción, debido a la extensión y al nivel de dificultad que esto podía suponer. De tal forma que el equipo del proyecto debía escoger algunas de las temáticas del curso y trabajar sobre ellas en el ejercicio gamificado.

Del total de ocho bloques temáticos, el equipo de proyecto seleccionó tres para ser abordadas con el ejercicio gamificado, cubriendo así el $38 \%$ del curso. Para la selección de estos bloques se siguieron tres pasos fundamentales: (1) durante la primera mitad del curso se profundizó en la observación en cuanto al uso de los materiales y de la herramienta ERP de SAP por parte de los estudiantes. (2) Posteriormente, se dedicó un espacio para el análisis del comportamiento de los estudiantes durante las clases en relación a su interacción con los recursos de cada bloque temático. (3) Finalmente, con toda la información recolectada, en el último tercio del curso se gamificaron las temáticas restantes del plan de estudio, las cuales se muestran en la Figura 2.

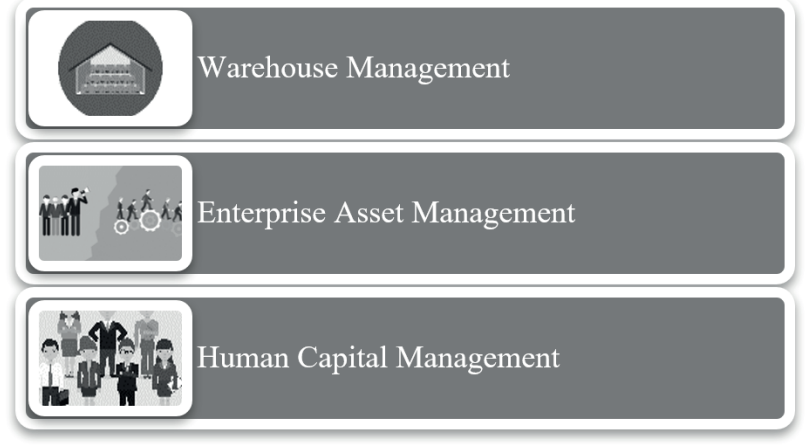

Figura 2. Bloques temáticos abordados en el ejercicio gamificado. Fuente: Autores. 
Nicolás Hernández-Durán, Martha Liliana Torres-Barreto, Mariana Acuña-Rangel

Classcraft como herramienta gamificada para la enseñanza de Integración de procesos con tecnología informática

Selección de la herramienta a utilizar para gamificar el curso

Para la selección de la herramienta de gamificación, el equipo de proyecto evaluó ocho diferentes opciones disponibles en la web. Los criterios de evaluación corresponden a una adaptación de la metodología propuesta por el Gobierno de Canarias en España (Sanabria, 2006), y que expresa que los ejercicios de gamificación se pueden evaluar en función de seis criterios. Estos se presentan en la Tabla 2.

Tabla 2

Criterios usados para la selección de la herramienta de gamificación

\begin{tabular}{|c|c|}
\hline Criterio & Descripción \\
\hline Accesibilidad & $\begin{array}{l}\text { Facilidad de adquisición e implementación de la } \\
\text { herramienta de manera gratuita. }\end{array}$ \\
\hline Atributos & $\begin{array}{l}\text { Tipos de aprendizaje que promueve o posibilita la } \\
\text { herramienta: activo/individualizado/colaborativo. }\end{array}$ \\
\hline Mecánica & $\begin{array}{l}\text { Diferentes reglas o estrategias que tiene la } \\
\text { herramienta que favorecen la competitividad, } \\
\text { fidelización y motivación de los usuarios. }\end{array}$ \\
\hline Narrativa & $\begin{array}{l}\text { Contexto e historia en la que se enmarca el } \\
\text { proceso de gamificación. }\end{array}$ \\
\hline Componentes & $\begin{array}{l}\text { Permiten al usuario involucrarse y llevar a cabo las } \\
\text { mecánicas. Se destacan: } \\
\text { - Avatares } \\
\text { - Mnsignias } \\
\text { - Misiones } \\
\text { - Recoloqueo de contenido } \\
\text { - Poder } \\
\text { - Tablero de clasificación } \\
\text { - Puneles } \\
\text { - Sorpresas } \\
\text { - Cuenta regresiva } \\
\text { - Barra de progreso }\end{array}$ \\
\hline
\end{tabular}

Tabla 3

Matriz de priorización

\begin{tabular}{|c|c|c|c|c|c|c|c|c|}
\hline \multirow[b]{2}{*}{ Herramientas } & Criterios & Accesibilidad & Atributos & Mecánica & Narrativa & Componentes & Aplicaciones & \\
\hline & Ponderación & $10 \%$ & $20 \%$ & $10 \%$ & $20 \%$ & $30 \%$ & $10 \%$ & $\begin{array}{c}\text { Total } \\
\text { Ponderado }\end{array}$ \\
\hline \multirow{2}{*}{ Brainscape } & Calificación & 2 & 1 & 2 & 1 & 2 & 3 & \multirow{2}{*}{1.7} \\
\hline & Total & 0.2 & 0.2 & 0.2 & 0.2 & 0.6 & 0.3 & \\
\hline \multirow{2}{*}{ Knowre } & Calificación & 3 & 2 & 1 & 1 & 2 & 3 & \multirow{2}{*}{1.9} \\
\hline & Total & 0.3 & 0.4 & 0.1 & 0.2 & 0.6 & 0.3 & \\
\hline \multirow{2}{*}{ Celibriti } & Calificación & 3 & 2 & 1 & 1 & 3 & 2 & \multirow{2}{*}{2.1} \\
\hline & Total & 0.3 & 0.4 & 0.1 & 0.2 & 0.9 & 0.2 & \\
\hline \multirow{2}{*}{$\begin{array}{c}\text { Minecraft: } \\
\text { Education edition }\end{array}$} & Calificación & 3 & 3 & 2 & 3 & 3 & 2 & \multirow{2}{*}{2.8} \\
\hline & Total & 0.3 & 0.6 & 0.2 & 0.6 & 0.9 & 0.2 & \\
\hline \multirow{2}{*}{ Pear Deck } & Calificación & 2 & 2 & 1 & 1 & 1 & 3 & \multirow{2}{*}{1.5} \\
\hline & Total & 0.2 & 0.4 & 0.1 & 0.2 & 0.3 & 0.3 & \\
\hline \multirow{2}{*}{ Kahoot } & Calificación & 3 & 2 & 1 & 1 & 3 & 3 & \multirow{2}{*}{2.2} \\
\hline & Total & 0.3 & 0.4 & 0.1 & 0.2 & 0.9 & 0.3 & \\
\hline \multirow{2}{*}{ Edmodo } & Calificación & 3 & 3 & 1 & 1 & 3 & 3 & \multirow{2}{*}{2.4} \\
\hline & Total & 0.3 & 0.6 & 0.1 & 0.2 & 0.9 & 0.3 & \\
\hline \multirow{2}{*}{ Calsscraft } & Calificación & 3 & 3 & 3 & 3 & 3 & 2 & \multirow{2}{*}{2.9} \\
\hline & Total & 0.3 & 0.6 & 0.3 & 0.6 & 0.9 & 0.2 & \\
\hline
\end{tabular}

Fuente: Autores.

\begin{tabular}{ll}
\hline Aplicaciones & $\begin{array}{l}\text { Herramientas TIC que soportan los diferentes } \\
\text { elementos de gamificación. }\end{array}$
\end{tabular}

Con base en estos criterios, se realizó una matriz de priorización en la que cada columna corresponde a uno de los criterios de la Tabla 2, y cada fila corresponde a las herramientas gamificadas que se están evaluando. La en una tabla de valores discretos, en la cual " 1 " equivale a poco significativo; " 2 ", a medianamente significativo, y " 3 ", a muy significativo. Adicionalmente, cada criterio está ponderado en razón de los resultados de la etapa de observación del grupo de estudiantes, de manera que se otorgó una mayor ponderación a los criterios que para los estudiantes resultan más interesantes. La ponderación efectuada en la Tabla 3 fue realizada por el equipo de proyecto, con base en su experiencia pedagógica, su conocimiento de la temática del curso y en los resultados de la etapa de observación.

Como resultado de esta priorización, Classcraft obtuvo una calificación de 2,9, superior a las otras herramientas evaluadas, de manera que fue la herramienta seleccionada para este ejercicio investigativo.

\section{Construcción del storytelling}

Con el propósito de estructurar una narrativa inmersiva e interesante para los usuarios, la historia que se narra está ambientada en una época futurista, en la cual son posibles los viajes a lo largo y ancho del universo. Además, los personajes protagonistas son piratas y cazarrecompensas interestelares, papeles que adoptarán cada uno de los estudiantes. El lugar donde
Fuente: Autores calificación que se otorga en cada celda está contenida 
Nicolás Hernández-Durán, Martha Liliana Torres-Barreto, Mariana Acuña-Rangel Classcraft como herramienta gamificada para la enseñanza de Integración de procesos con tecnología informática

se desarrollan los hechos es un planeta llamado Fearless, en donde coexisten algunas comunidades, unas más evolucionadas que otras, las cuales presentan una serie de problemáticas relacionadas con la integración de procesos mediante tecnología informática, para lo cual deciden enviar una señal al espacio pidiendo ayuda a cualquier forma de vida que tuviera el conocimiento suficiente para solucionar sus problemas y así obtener una recompensa. Los protagonistas del juego se verán involucrados en diferentes escenarios donde tendrán que dar solución a algunos casos de estudio propuestos por la plataforma ERP. Los alumnos que cumplan con las actividades relacionadas tendrán la oportunidad de avanzar en la historia, obtener experiencia y así alcanzar nuevos niveles que les abrirán las puertas a nuevas posibilidades y retos más complejos.

Para el desarrollo de esta narrativa se utilizó la metodología propuesta por Wang et al. (2018) en su artículo Human-Guided Evolutionary Story Narration.

\section{Selección de los comportamientos a promover en el aula}

Uno de los aspectos clave de la gamificación es que facilita, mediante el juego, la promoción de comportamientos entre los jugadores (Bozkurt y Durak, 2018). Algunos autores están de acuerdo en que las estrategias de enseñanza permiten coordinar y aplicar habilidades (Nisbet y Shucksmith, 2017), y se vinculan con el aprendizaje significativo y con el aprender a aprender. En la academia esto se ha empleado, por ejemplo, para conseguir solidaridad entre compañeros, cooperación, trabajo en equipo, etc.

En el diseño de comportamientos para promover en el aula se tuvieron en cuenta algunos tipos de estrategias de aprendizaje, tales como:

- Estrategias de búsqueda, recolección y selección de información: integran todo lo referente a la localización, recolección y selección de información. El sujeto debe aprender, para ser aprendiz estratégico, cuáles son las fuentes de información y cómo acceder a ellas para disponer de esta. Debe aprender, también, mecanismos y criterios para seleccionar la información pertinente.

Con base en esto, y con algunas experiencias identificadas mediante observación en las aulas del curso de Integración de procesos con tecnología informática, el equipo de proyecto desarrolló los comportamientos, a los cuales se les asignó una puntuación positiva o negativa en función del contexto en que se enmarca la herramienta anteriormente seleccionada; es decir, a aquellos comportamientos que se querían promover en el aula se les asignó una cantidad de puntos de experiencia, la cual iba a ser proporcionada a quienes cumplieran con lo escrito. Por otro lado, a aquellos comportamientos que no se querían promover se les asignó una cantidad de puntos de salud, los cuales iban a ser restados a los estudiantes que cometieran alguna falta de las allí mencionadas.

A continuación, se presentan en las Tablas 4 y 5 los comportamientos desarrollados.

Tabla 4

Comportamientos para promover en las aulas

\begin{tabular}{cl}
\hline \multicolumn{2}{c}{ Comportamientos a promover } \\
\hline $\mathbf{X P}$ & \multicolumn{1}{c}{ Descripción } \\
\hline $\mathbf{5 0}$ & $\begin{array}{l}\text { Entregar material adicional/complementario sobre un } \\
\text { tema de clase }\end{array}$ \\
$\mathbf{5 0}$ & $\begin{array}{l}\text { Exponer voluntariamente en clase un recurso } \\
\text { relacionado con el tema de clase }\end{array}$ \\
$\mathbf{2 0}$ & $\begin{array}{l}\text { Responder correctamente una pregunta que la profesora } \\
\text { hace en clase }\end{array}$ \\
$\mathbf{1 0 0}$ & $\begin{array}{l}\text { Completar todo el caso de estudio durante la clase } \\
\mathbf{7 5}\end{array}$ \\
$\begin{array}{l}\text { Ayudar a otro estudiante en la solución del caso de } \\
\text { estudio }\end{array}$ \\
$\mathbf{3 0}$ & $\begin{array}{l}\text { Encontrar la solución técnica a un problema del caso de } \\
\mathbf{5 0}\end{array}$ \\
$\mathbf{5 0}$ & Encontrar un artículo o recurso relacionado con la lección \\
\hline
\end{tabular}

Fuente: Autores.

Tabla 5

Comportamientos que no se van a promover en las aulas

\begin{tabular}{cl}
\hline & \multicolumn{1}{c}{ Comportamientos a promover } \\
\hline XP & \multicolumn{1}{c}{ Descripción } \\
\hline-20 & No hacer tu parte del trabajo de grupo \\
$-\mathbf{1 5}$ & Preguntar por conceptos que ya se han dado antes \\
$-\mathbf{4 0}$ & Bloqueo de la cuenta proporcionada por el ERP \\
$-\mathbf{2 0}$ & Comer en clase \\
$-\mathbf{1 0}$ & Quedarse dormido en clase \\
$-\mathbf{1 0}$ & Preguntar a la docente sin antes buscar en el centro de \\
$\mathbf{- 1 2}$ & Lleluciones del ERP \\
\hline
\end{tabular}

Fuente: Autores.

\section{Asignación de poderes y eventos aleatorios}

A pesar de que la copia o plagio siempre ha estado presente en las aulas (Comas-Forgas y Sureda-Negre, 2010), es uno de los comportamientos que como docentes deseamos evitar, por lo que incentivamos a los estudiantes a que actúen con sinceridad y honestidad. En los últimos años el fenómeno ha adquirido una nueva dimensión, también gracias a internet (Comas-Forgas y Sureda-Negre, 2010). Teniendo en cuenta lo anterior, el equipo de proyecto identificó un uso potencial de uno de los recursos que ofrece la plataforma de Classcraft: los poderes. 
Nicolás Hernández-Durán, Martha Liliana Torres-Barreto, Mariana Acuña-Rangel

Classcraft como herramienta gamificada para la enseñanza de Integración de procesos con tecnología informática

Para la selección de los poderes que iban a tener los avatares de los estudiantes, se realizó un análisis de los poderes predefinidos con los que contaba la plataforma de Classcraft. Con base en ello, se realizaron las modificaciones que se consideraron pertinentes teniendo en cuenta algunas técnicas de "plagio" que utilizan comúnmente los estudiantes en las aulas de clase, ya que la idea de estos poderes era brindarles a los participantes una forma de alcanzar sus metas de manera sencilla, pero sobre todo legal, dentro del contexto en que se desarrolla la experiencia. En las Tabla 6, Tabla 7 y Tabla 8 se presentan las listas de poderes según el rol, a los cuales los estudiantes podían acceder a medida que subían de nivel sus avatares.

Tabla 6

Poderes de la clase MAGO

\begin{tabular}{lc}
\hline \multicolumn{1}{c}{ Descripción } & $\begin{array}{c}\text { Costo } \\
\text { de PA }\end{array}$ \\
\hline $\begin{array}{l}\text { Todos los miembros del equipo, excepto los magos, giran } \\
7 \text { puntos de acción }\end{array}$ & 35 \\
$\begin{array}{l}\text { El mago puede visitar a otro equipo durante dos minutos } \\
\text { El mago puede comer en clase }\end{array}$ & 5 \\
$\begin{array}{l}\text { El mago evita la pérdida de puntos de salud } \\
\begin{array}{l}\text { Un compañero caído puede volver a tirar el dado maldito, } \\
\text { pero debe aceptar un nuevo resultado }\end{array}\end{array}$ & 10 \\
$\begin{array}{l}\text { El mago gana media hora adicional para superar un } \\
\text { examen }\end{array}$ & 25 \\
$\begin{array}{l}\text { Un compañero de equipo recarga todos sus puntos de } \\
\text { acción }\end{array}$ & 40 \\
$\begin{array}{l}\text { Todos los miembros del equipo reciben una pista sobre } \\
\text { una pregunta del examen }\end{array}$ & 40 \\
$\begin{array}{l}\text { Todos los miembros del equipo ganan media hora para } \\
\text { superar un examen }\end{array}$ & 55 \\
\hline
\end{tabular}

Fuente: Autores.

Tabla 7

Poderes de la clase GUERRERO

\begin{tabular}{lc}
\hline \multicolumn{1}{c}{ Descripción } & $\begin{array}{c}\text { Costo } \\
\text { de PA }\end{array}$ \\
\hline $\begin{array}{l}\text { El guerrero puede recibir hasta 10 de daño en lugar de su } \\
\text { compañero de equipo }\end{array}$ & 10 \\
$\begin{array}{l}\text { El guerrero gana } 1 \text { punto de salud por cada nivel que } \\
\text { tenga, pero siempre gana al menos 5 }\end{array}$ & 10 \\
$\begin{array}{l}\text { El guerrero puede comer en clase } \\
\text { El guerrero puede recibir hasta } 20 \text { de daño en lugar de }\end{array}$ & 5 \\
$\begin{array}{l}\text { su equipo } \\
\text { El guerrero puede entregar la tarea un día más tarde }\end{array}$ & 20 \\
$\begin{array}{l}\text { El guerrero obtiene una pista sobre una pregunta del } \\
\text { examen }\end{array}$ & 20 \\
$\begin{array}{l}\text { El guerrero puede recibir hasta 30 de daño en lugar de su } \\
\text { compañero de equipo }\end{array}$ & 20 \\
$\begin{array}{l}\text { Todos los miembros del equipo reciben una pista sobre } \\
\text { una pregunta del examen }\end{array}$ & 50 \\
\begin{tabular}{l} 
El guerrero puede usar sus apuntes en el examen \\
\hline
\end{tabular} & 70 \\
\hline
\end{tabular}

Fuente: Autores.
Tabla 8

Poderes de la clase SANADOR

\begin{tabular}{lc}
\multicolumn{1}{c}{ Descripción } & Costo de PA \\
\hline $\begin{array}{l}\text { Un compañero obtiene } 10 \text { puntos de salud } \\
\text { El sanador puede revisar su teléfono durante } 5\end{array}$ & 45 \\
minutos en el examen & 15 \\
$\begin{array}{l}\text { Durante un examen el sanador puede preguntarle al } \\
\text { profesor si su respuesta a una pregunta es correcta }\end{array}$ & 10 \\
$\begin{array}{l}\text { Un compañero obtiene } 20 \text { puntos de salud } \\
\text { El sanador recibe 10 puntos de salud }\end{array}$ & 20 \\
$\begin{array}{l}\text { Cuando un compañero de equipo muere, evita } \\
\text { todas las penalizaciones y resucita con un punto de } \\
\text { salud }\end{array}$ & 20 \\
$\begin{array}{l}\text { Un compañero obtiene } 30 \text { puntos de salud } \\
\text { Todos los miembros del equipo, excepto el sanador, }\end{array}$ & 20 \\
ganan 15 puntos de salud & 30 \\
$\begin{array}{l}\text { El sanador puede usar una ayuda pedagógica en el } \\
\text { examen }\end{array}$ & 50 \\
\hline $\begin{array}{l}\text { Fuente: Autores. } \\
\end{array}$ &
\end{tabular}

Ejecución y resultados de la experiencia gamificada

Con base en lo descrito, se ejecutaron dos actividades que permitieron cerrar el ciclo de diseño de la experiencia gamificada. Estas son: validación de la funcionalidad y juego de prueba.

La validación de la funcionalidad y el juego de prueba se realizaron con la totalidad de los estudiantes de los dos cursos de la electiva. Se realizaron los recorridos por 4 misiones (ver Figura 3), durante un total de 4 semanas de trabajo, con una intensidad de 4 horas por semana. La docente de la materia, apoyada por los dos auxiliares, guiaba la clase siguiendo los contenidos que fueron cargados totalmente en Classcraft.

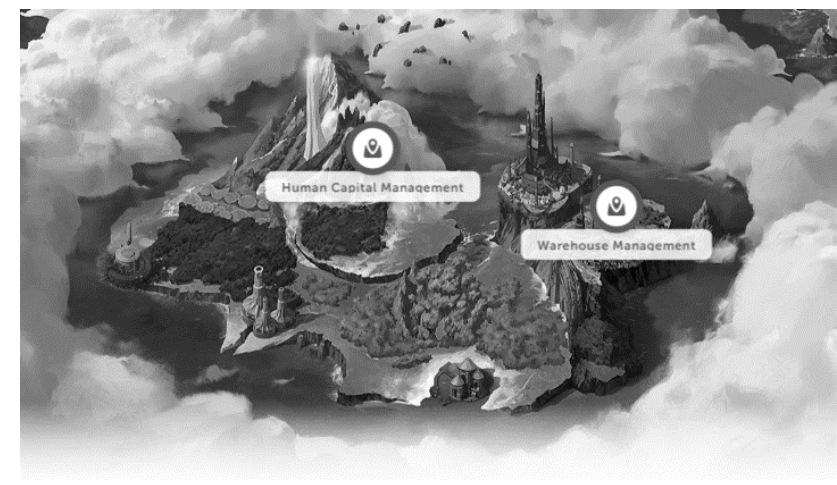

Figura 3. Mapa de misiones Classcraft. Fuente: Adaptación de la plataforma Classcraft.

Los estudiantes, cada uno en su ordenador, escogieron su avatar, y de acuerdo con él, tenían poderes diversos. Al inicio de la clase la docente proporcionó una explicación general del contenido de esa sesión, y a continuación, los estudiantes ingresaron con su avatar a las misiones que debían completar. En cada misión se encontraba 
Nicolás Hernández-Durán, Martha Liliana Torres-Barreto, Mariana Acuña-Rangel Classcraft como herramienta gamificada para la enseñanza de Integración de procesos con tecnología informática

un storytelling que describía las tareas que iban a tener que ejecutar en esa misión, proporcionaba un material explicativo de la temática relacionada con la asignatura, y brindaba un espacio para subir actividades, tan pronto como el estudiante iba terminando su ejercicio de forma individual (ver Figura 4).

Asignación de Gina Alexandra Alza Avila

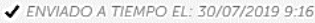

(2itorres_8. WM_casestudy_1.doc

\pm DESCARGAR TODO

\section{ANULAR ENVIO}

Figura 4. Asignación entregada a tiempo en la plataforma. Fuente: Adaptación de la plataforma de Classcraft.

A medida que los estudiantes iban desarrollando las diferentes actividades propuestas en el marco de las temáticas de integración de procesos con tecnología informática, la herramienta gamificada les brindaba puntos de experiencia por actividad y por cumplimiento de objetivos, lo cual les permitía evolucionar sus avatares y así obtener poderes que les fueran de utilidad en el desarrollo del curso (ver Figura 5).

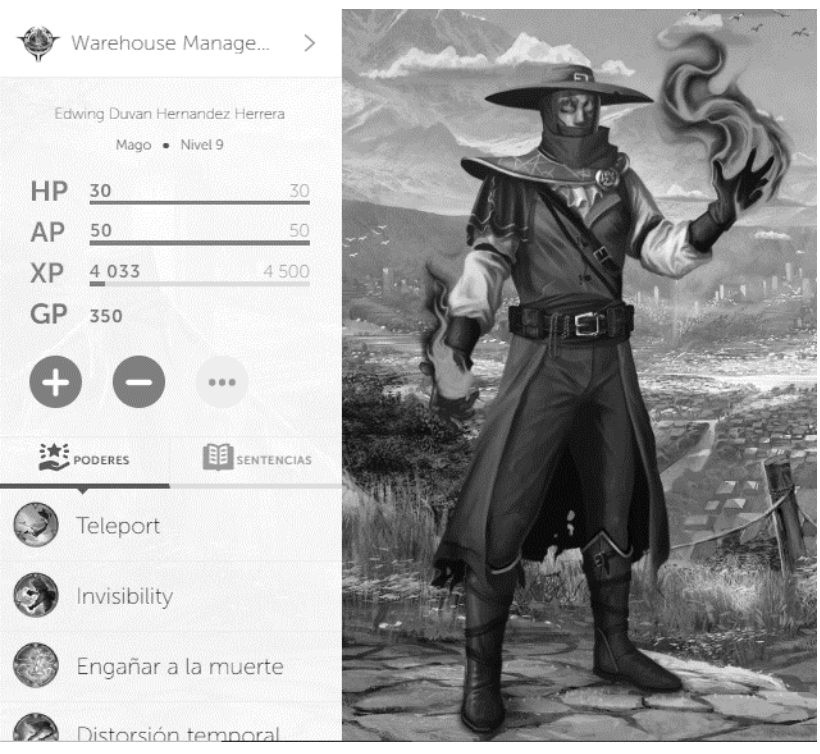

Figura 5. Estadísticas de personaje. Fuente: Adaptación de interfaz de juego Classcraft.

Eventualmente, durante el transcurso de la clase, la docente generaba eventos aleatorios que podrían ser positivos o negativos, entre ellos: (1) Conocimiento peligroso: todos los estudiantes de un equipo al azar deberán responder una pregunta correctamente, cada estudiante que lo haga bien obtendrá 150 puntos de experiencia, quien lo haga mal perderá 20 puntos de salud. (2) Poción de silencio: todos los magos deben guardar estricto silencio durante toda la clase. Si no lo hacen, perderán 5 puntos de salud y 15 de experiencia. Y (3) Modales cortesanos: todos los estudiantes deben referirse a sus compañeros como "Milord" o "Milady" cuando les dirijan la palabra durante esa clase. Las reacciones de los estudiantes eran variadas, y correspondían al efecto positivo o negativo que tales eventos aleatorios creaban sobre la bolsa de puntos de cada estudiante (ver Figura 6).

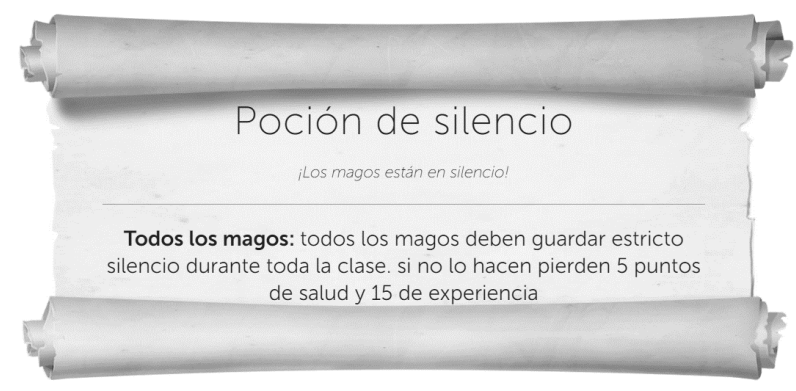

Figura 6. Eventos aleatorios: "Poción de silencio". Fuente: Adaptación de plataforma Classcraft.

Al finalizar las 4 semanas de trabajo con Classcraft, se realizó una evaluación convencional sobre las competencias que se esperaba que los estudiantes tuvieran al finalizar el curso. Ningún estudiante obtuvo calificación por debajo del mínimo necesario para aprobar.

Una vez repartidos los resultados de las evaluaciones finales, se pidió a los estudiantes participar de forma voluntaria en la fase de evaluación de la experiencia, y para ello se les proporcionó un enlace que daba paso a un cuestionario de Google.

De los 43 estudiantes, 40 quisieron participar de forma anónima y voluntaria en la encuesta. Los resultados permiten deducir que cerca del $60 \%$ de los estudiantes se implicaron en un grado ALTO o MUY ALTO en el uso de la herramienta, y solo un $5 \%$ se implicó en un grado BAJO. El sistema de puntos y el avatar fueron los elementos gamificados mejor valorados por los estudiantes que participaron en el ejercicio. Los eventos aleatorios y las insignias fueron, por su parte, los elementos peor valorados por los estudiantes. Llama especialmente la atención el hecho de que el factor competitivo de la experiencia gamificada fue el mejor valorado por el conjunto de participantes, lo cual está en línea con diversos autores que señalan que la competición ha sido siempre un elemento estimulante. Siempre cabe una dosis de este sentimiento en la naturaleza humana. Se puede ser más o menos competitivo, y existe una pedagogía de la bondad en la competición o una manera sana, útil y práctica de hacer que los alumnos participen (Foncubierta y Rodríguez, 2014). 
Nicolás Hernández-Durán, Martha Liliana Torres-Barreto, Mariana Acuña-Rangel

Classcraft como herramienta gamificada para la enseñanza de Integración de procesos con tecnología informática

Frente a la cuestión de si los estudiantes prefieren una clase gamificada frente a una no gamificada, si los contenidos temáticos fueran los mismos, el 77,5\% contestó que prefiere la clase gamificada.

Las recompensas fueron atractivas para el $47,5 \%$ de los estudiantes, mientras que las misiones lo fueron para el $50 \%$ de la clase. Para el 17,5\% de los estudiantes, tanto los eventos como las insignias fueron un aspecto de muy bajo interés.

\section{Discusión y conclusiones}

El ejercicio gamificado realizado en la clase Integración de procesos con tecnologías de la información tuvo una aceptación elevada entre los estudiantes que realizaron el proceso de validación de funcionalidad y el juego de prueba. La mayoría de estos estudiantes manifiesta que prefiere una clase gamificada, lo cual da respuesta a la pregunta de investigación formulada para este estudio, ya que el $77,5 \%$ de estudiantes prefiere la gamificación como herramienta didáctica que facilita el aprendizaje experiencial.

En cuanto a los elementos usados en esta estrategia gamificada, algunos son mejor valorados que otros por los participantes. Esto puede deberse a la misma percepción y sentido de competencia que tiene el juego implementado en Classcraft. Si en el escenario de competición un estudiante estuvo en la parte alta de la tabla de puntos, ese estudiante tiene tendencia a calificar positivamente la experiencia, frente a otro que estuvo en la parte más baja de la tabla. Por otra parte, la evaluación de los eventos aleatorios fue en realidad muy negativa. Esto puede obedecer a la valoración que los seres humanos damos al factor aleatorio, ya que deseamos tener bajo nuestro control los eventos tanto positivos como negativos que nos ocurran, en lugar de dejar al "azar" las cosas que sucedan y que nos puedan afectar.

Seleccionar la funcionalidad de forma totalmente aleatoria sería ignorar el modelo de usuario. Pero si no hay aleatoriedad, las funcionalidades que implementan nuevos factores de motivación para este usuario nunca serán probadas (Monterrat et al., 2014).

Por el contrario, el hecho de contar con un avatar o personaje que represente al estudiante en el juego llena de emoción cada sesión. Esto puede estar asociado a la teoría del sujeto virtual, de identidades en "la red" que poco tienen que ver con la identidad real (Turkle, 1996). Los avatares permiten que cada sujeto se presente de una manera ideal, permiten construir nuevos yoes, nuevas identidades personales a través de una interacción social creada en el entorno gamificado de la clase. Los personajes virtuales tienen poderes, ganan y pierden dinero, ascienden y descienden en el estatus social. Un personaje virtual también puede morir. A diferencia del entorno gamificado, la vida real no permite que esto ocurra sin mayores consecuencias. Esto puede ser precisamente lo que hace que sean tan llamativos los avatares dentro de un entorno gamificado. Como investigadores vemos un potencial amplio para seguir ahondando en nuestros trabajos a partir de esta experiencia inicial. Uno de los primeros frentes sería completar toda la asignatura con el ejercicio de gamificación y volver a evaluar la percepción de los usuarios.

Otro frente debería estar dedicado a las diferencias que puede haber en el aprendizaje de estudiantes que usan la herramienta gamificada, frente a otro grupo de control que no la usa. Finalmente, es importante resaltar el valor que puede aportar al aprendizaje, el uso de entornos gamificados y de micromundos virtuales que favorecen la transmisión del conocimiento y además despierta el interés de los estudiantes por aprender incluso en temas en los que es difícil generarlo (TorresBarreto, 2014). La aplicación de este ejercicio gamificado en los cursos de integración de procesos con tecnología informática facilitó a los estudiantes el aprendizaje y la apropiación de herramientas que son imprescindibles en la industria para la gestión de la información de manera óptima. Gracias a que Classcraft es una herramienta muy maleable y adaptable a diferentes entornos educativos, fue posible hacer divertidas las clases teórico-prácticas y fomentar la participación de los alumnos en las aulas. Sin lugar a duda, el sujeto y la relación social virtual debe ser objeto de estudio, en cuando las comunidades virtuales como forma de expresión de nuestra sociabilidad marcan una tendencia a futuro.

\section{Referencias}

Acosta-Medina, J. K., Torres-Barreto, M. L. y AlvarezMelgarejo, M. (2020). Literature Mapping About Gamification in the Teaching and Learning Processes. Revista ESPACIOS, 41(11), 26. https://www. revistaespacios.com/a20v41n11/20411126.html

Acosta-Medina, J. K., Torres-Barreto, M. L., AlvarezMelgarejo, M. y Paba-Medina, M. C. (2020). Gamificación en el ámbito educativo: Un análisis bibliométrico. I+D Revista de Investigaciones, 15(1), 30-39.https://doi.org/10.33304/revinv.v15n1-2020 003

Aji, T. P. y Napitupulu, T. A. (2018). Effect of Gamification on E-Learning to Support Learning Achievement and Learning Motivation. Journal of Theoretical and Applied Information Technology.

Berchet, C. y Habchi, G. (2005). The Implementation and Deployment of an ERP System: An Industrial Case Study. Computers in industry, 56(6), 588-605.

Bozkurt, A. y Durak, G. (2018). A Systematic Review of Gamification Research: In pursuit of homo ludens. 
Nicolás Hernández-Durán, Martha Liliana Torres-Barreto, Mariana Acuña-Rangel Classcraft como herramienta gamificada para la enseñanza de Integración de procesos con tecnología informática

International Journal of Game-Based Learning, 8(3), 15-33. https://doi.org/10.4018/ijgbl.2018070102

Burke, B. (2016). Gamify (1. ${ }^{\mathrm{a}}$ ed.). Taylor \& Francis Group. https://doi.org/10.4324/9781315230344

Campos, A. (2010). Neuroeducación: Uniendo las neurociencias y la educación en la búsqueda del desarrollo humano.La educ@ación, 143, 1-14.

Comas-Forgas, R. y Sureda-Negre, J. (2010). Academic Plagiarism: Explanatory Factors from Students' Perspective. Journal of Academic Ethics, 8(2), 217232. https://doi.org/10.1007/s10805-010-9121-0

Davenport, T. y Short, J. (1990). The New Industrial Engineering: Information Technology and Business Process Redesign. Sloan Management Review.

Dunbar, K. N. (2008). Arts Education, the Brain, and Language. En C. Asbury y B. Rich (eds.), Learning, Arts, and the Brain. The Dana Consortium Report on Arts and Cognition (pp. 81-92). Dana Press.

Estupiñan Ricardo, J., Cherrez Cano, I., Intriago Alcívar, G. y Torres Vargas, R. (2016). Neurociencia cognitiva e inteligencia emocional. La gestión pedagógica en el contexto de la Formación Profesional.Didasc@ lia: Didáctica y Educación, 12(4), 207-214.

Foncubierta, J. y Rodríguez, C. (2014). Didáctica de la gamificación en la clase de español. Edinumen.

Frost, J. y Eden, A. (2014). The Effect of Social Sharing Games and Game Performance on Motivation to Play Brain Games. En B. Schouten, S. Fedtke, M. Schijven, M. Vosmeer y A. Gekker (eds.), Games for Health 2014 (pp. 48-55). Springer Vieweg.

Glover, I. (2013). Play As You Learn: Gamification as a Technique for Motivating Learners. Proceedings of EdMedia 2013-World Conference on Educational Media and Technology, 1999-2008.

Hardiman, M. M. (2003). Connecting Brain Research with Effective Teaching: The Brain-Targeted Teaching Model. Scarecrow Press.

Ito, M. (2000). Mechanisms of Motor Learning in the Cerebellum. Brain research, 886(1-2), 237-245.

Kapp, K. M. (2012). Research Says...Games are Effective for Learning. En The gamification of learning and instruction: game-based methods and strategies for training and education (75-103). John Wiley \& Sons.

Klock, A. C. T., Gasparini, I. y Pimenta, M. (2019). UserCentered Gamification for E-Learning Systems: A Quantitative and Qualitative Analysis of its
Application. Interacting with Computers, 31(5), 425445. https://doi.org/10.1093/iwc/iwz028

Lobo-Rueda, M. A., Paba-Medina, M. C. y Torres-Barreto, M. L. (2020). Análisis descriptivo de experiencias gamificadas para enseñanza y aprendizaje en educación superior en ingeniería. Revista ESPACIOS, 41(16), 21. https://www.revistaespacios.com/ a20v41n16/20411621.html

Marache-Francisco, C. y Brangier, E. (2014). The Gamification Experience: UXD with a Gamification Background. En K. Blashki y P. Isaias (eds.), Emerging Research and Trends in Interactivity and the HumanComputer Interface (pp. 205-223). IGI Global. https:// doi.org/10.4018/978-1-4666-4623-0.ch010

Monterrat, B., Lavoué, É. y George, S. (2014). Motivation for Learning: Adaptive Gamification for WebBased Learning Environments. CSEDU 2014 Proceedings of the 6th International Conference on Computer Supported Education, 117-125. https://doi. org/10.5220/0004848101170125

Morales, D. y Alfonso, Y. M. (2006). La importancia de promover en el aula estrategias de aprendizaje para elevar el nivel académico en los estudiantes de Psicología. Revista Iberoamericana de Educación, 40(1), 1-17. https://doi.org/10.35362/rie4012532

Nikolopoulos, K., Metaxiotis, K., Lekatis, N. y Assimakopoulos, V. (2003). Integrating Industrial Maintenance Strategy into ERP. Industrial Management \& Data Systems, 103(3), 184-191. https://doi.org/10.1108/02635570310465661

Nisbet, J. y Shucksmith, J. (2017). Learning Strategies (1. ${ }^{\text {a }}$ ed.). Taylor \& Francis Group. https://doi. org/10.4324/9781315188652

Rojas-López, A., Rincón-Flores, E. G., Mena, J., GarcíaPeñalvo, F. J. y Ramírez-Montoya, M. S. (2019). Engagement in the Course of Programming in Higher Education Through the Use of Gamification. Universal Access in the Information Society, 18(3), 583597. https://doi.org/10.1007/s10209-019-00680-z

Sanabria, A. (2006). Las TIC en el sistema escolar de Canarias: Los programas institucionales de innovación educativa para la integración curricular de las Tecnologías de la Información y la Comunicación. Revista Latinoamericana de Tecnología Educativa-RELATEC, 5(2), 191-202.

Sousa, D. A. (2011). How The Brain Learns. Corwin Press.

Torres-Barreto, M. L. (2014). Los micro mundos virtuales como apoyo al proceso de aprendizaje en la 
Nicolás Hernández-Durán, Martha Liliana Torres-Barreto, Mariana Acuña-Rangel

Classcraft como herramienta gamificada para la enseñanza de Integración de procesos con tecnología informática

educación media. Encuentro Internacional de Educación en Ingeniería ACOFI 2014.

Torres-Barreto, M. L. (2018). Herramienta didáctica motivacional basada en gamificación y apoyada en TIC para adquirir y aplicar competencias transversales en estudiantes de ingeniería: MOTIVATIC (hal-02166319). https://hal.archives-ouvertes.fr/hal02166319/

Turkle, S. (1996). Who Am We? Wired Magazine, 4(1).

Wang, K., Bui, V., Petraki, E. y Abbass, H. A. (2018). Human-Guided Evolutionary Story Narration. IEEE Access, 6(3), 13783-13802. https://doi.org/10.1109/ ACCESS.2018.2797879

Werbach, K. y Hunter, D. (2012). Level 5: Game Changer: Six steps to gamification. En For the Win: How Game Thinking Can Revolutionize Your Business. Wharton Digital Press. (85-102).

Zichermann, G. y Cunningham, C. (2011). Gamification by Design: Implementing Game Mechanics in Web and Mobile Apps (1. ${ }^{\mathrm{a}}$ ed.). O'Reilly Media. 